\title{
The Functional Renormalization Group and $O(4)$ scaling
}

\author{
B. Stokić ${ }^{1}$ B. Friman, ${ }^{1}$ and K. Redlich ${ }^{2,3}$ \\ ${ }^{1}$ Gesellschaft für Schwerionenforschung, GSI, D-64291 Darmstadt, Germany \\ ${ }^{2}$ Institute of Theoretical Physics, University of Wroclaw, PL-50204 Wroctaw, Poland \\ ${ }^{3}$ Institut für Kernphysik, Technical University Darmstadt, D-64289 Darmstadt, Germany
}

(Dated: October 30, 2018)

\begin{abstract}
The critical behavior of the chiral quark-meson model is studied within the Functional Renormalization Group (FRG). We derive the flow equation for the scale dependent thermodynamic potential at finite temperature and density in the presence of a symmetry-breaking external field. Within this scheme, the critical scaling behavior of the order parameter, its transverse and longitudinal susceptibilities as well as the correlation lengths near the chiral phase transition are computed. We focus on the scaling properties of these observables at non-vanishing external field when approaching the critical point from the symmetric as well as from the broken phase. We confront our numerical results with the Widom-Griffiths form of the magnetic equation of state, obtained by a systematic $\epsilon-$ expansion of the scaling function. Our results for the critical exponents are consistent with those recently computed within Lattice Monte-Carlo studies of the $O(4)$ spin system.
\end{abstract}

PACS numbers:

\section{INTRODUCTION}

Understanding the phase structure and the critical properties of strongly interacting matter is one of the central problems addressed in studies of QCD thermodynamics. Lattice Gauge Theory (LGT) calculations at finite temperature show a clear separation between the confined (hadronic) and deconfined (quark-gluon plasma) phases [1].

Universality arguments imply that two-flavor QCD exhibits a second order chiral phase transition belonging to the same universality class as the $O(4)$ spin system in three dimensions [2, 3, 4, 5, 6, 6, 7, 8]. Consequently, the long-range properties of the chiral phase transition can be explored within effective models of the same universality class, independent of the specific dynamics.

One such model is the chiral quark-meson model, which often is used as an effective realization of the lowenergy sector of QCD [9]. Thus, by studying the thermodynamics of this model near the phase transition, one can explore the leading singularities of thermodynamic quantities in two-flavor QCD at the second order chiral phase transition. In other words, we can find the universal properties of dynamical chiral symmetry breaking at finite temperature and/or density. However, at this point we stress that a description of the QCD phase transition within effective models is subject to limitations. First, in chiral effective models one cannot address the deconfinement phenomenon in QCD and second, one cannot obtain quantitative information on the thermodynamics outside the chiral critical region. More generally, nonuniversal quantities depend on the specific dynamics of the theory and can therefore not be computed reliably within effective models.

As a rule, phase transitions and critical phenomena are related with singular behavior of some susceptibility which in turn provide a measure of the fluctuations of a physical quantity. At a second order phase transition, there are long-range critical correlations that appear due to the presence of massless modes. The long-range correlations imply divergent susceptibilities and consequently large fluctuations.

In the mean field approximation, which is often used to describe the chiral phase transition [10, 11, 12], one fails to obtain a quantitatively correct description of critical phenomena, since the influence of fluctuations and non-perturbative effects near the phase transition are neglected. However, methods based on the renormalization group (RG) can account for both of these important effects (see e.g. 13]). The advantage of the RG method is that it can describe physics across different momentum scales. In particular, within the RG framework one can capture the dynamics of the long-range fluctuations near the critical point. Wilsonian RG techniques, such as the functional renormalization group (FRG), are particularly useful in describing phase transitions 14]. One of the important results of RG theory is that it provides a rationale for universality, i.e., the fact that models can be grouped into universality classes that, depending only on the dimension of the system and the symmetry of the order parameter, show the same critical behavior.

In this paper we use the FRG method in the quarkmeson model to explore the universal properties of the chiral phase transition. This model has been studied using different RG approaches [9, 15, 16, 17, 18] both in vacuum as well as in medium, at finite temperature and chemical potential. Various critical exponents have also been computed [19, 20, 21, 22]. In this paper we go beyond previous RG studies of this model. We analyze the critical region in the broken and in the symmetric phase close to the critical point and discuss the scaling functions for different physical quantities as well as the magnetic equation of state [23]. Furthermore, we compute the critical exponents for the order parameter and its transverse and longitudinal susceptibilities as well as for the correlation lengths. We also calculate the effec- 
tive critical exponents [24] in the presence of the chiral symmetry breaking field. Finally, we explore the scaling behavior of different ratios of susceptibilities and the order parameter [25].

We also show that within the FRG method, with a suitable truncation of the FRG flow equations, the quarkmeson model exhibits a universal behavior that is in quantitative agreement with that recently found in lattice calculations of the $O(4)$ spin system in three dimensions [26].

The paper is organized as follows: In Sec. [II we introduce the chiral quark-meson model and the FRG method. The flow equations for the scale dependent thermodynamic potential at finite temperature and chemical potential are also derived in this Section. In Sec. III we discuss the universal scaling properties of various physical quantities and extract their critical exponents. In Sec. IV we summarize our results and discuss their relevance.

\section{THE CHIRAL QUARK-MESON MODEL AND THE FRG FLOW EQUATION}

We employ the chiral quark-meson model to explore the scaling properties and the critical equation of state within the FRG method. This model is relevant for studying strongly interacting hot and dense matter with two degenerate light-quark flavors, since it is expected to belong to the same universality class as QCD at the chiral phase transition.

The chiral quark-meson model represents an effective low-energy realization for spontaneous chiral symmetry breaking at the intermediate momentum scale $4 \pi f_{\pi} \approx 1$ $\mathrm{GeV}$. One can view this model as an effective model of QCD, where the gluon degrees of freedom have been integrated out. Consequently, the model does not describe the confinement-deconfinement transition.

The Lagrangian density of the chiral quark-meson model is given by

$$
\mathcal{L}=\frac{1}{2}\left(\partial_{\mu} \phi\right)^{2}+\bar{q} i \not \partial q+g \bar{q} M q+U(\sigma, \vec{\pi}),
$$

where the $O(4)$ representation of the meson fields is $\phi=(\sigma, \vec{\pi})$ and the corresponding $S U(2)_{L} \otimes S U(2)_{R}$ chiral representation is given by $M=\sigma+i \vec{\tau} \cdot \vec{\pi} \gamma_{5}$. There are $N_{f}^{2}=4$ mesonic degrees of freedom coupled to $N_{f}=2$ quark flavors $q$.

The potential $U(\sigma, \vec{\pi})$ is given by

$$
U(\sigma, \vec{\pi})=\frac{1}{2} m^{2} \phi^{2}+\frac{\lambda}{4} \phi^{4}-c \sigma .
$$

In vacuum, the $O(4)$ symmetry of the Lagrangian (1) is, for $m^{2}<0$, spontaneously broken to $O(3)$. This leads to a nonvanishing scalar condensate [44] $\langle\sigma\rangle=f_{\pi}$. The explicit symmetry breaking term $c \sigma$ in the potential gives the pion a mass $m_{\pi}$. At the three level, $c_{0}=f_{\pi} m_{\pi}^{2}$ yields

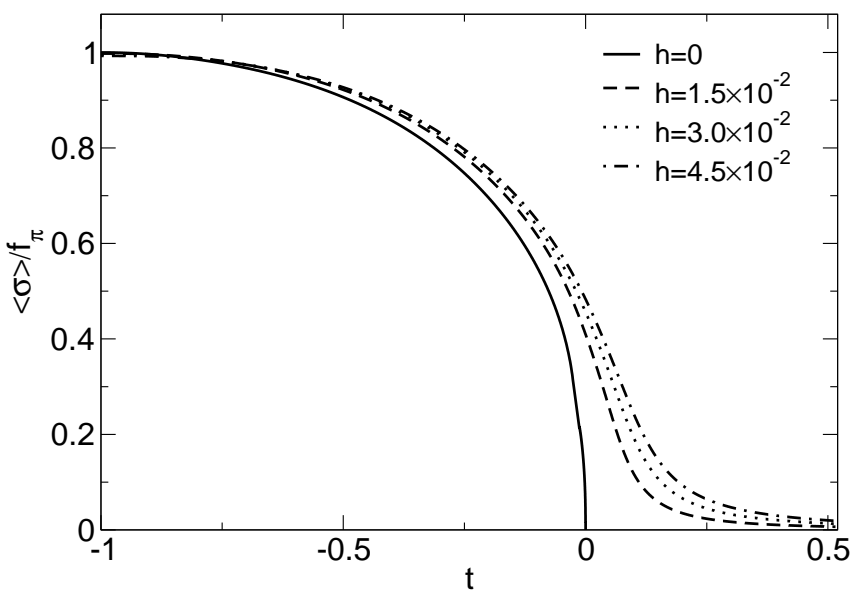

FIG. 1: The order parameter as a function of the reduced temperature $t$ for several values of the external field $h$.

the physical pion mass in vacuum. For convenience we introduce a dimensionless parameter $h=c / c_{0}$, the reduced external field, as a measure for the strength of the symmetry breaking term. In a medium, the chiral symmetry of the Lagrangian is restored, leading to a vanishing scalar condensate at some critical temperature and/or density.

To explore the critical properties near the chiral phase transition we use the Functional Renormalization Group. The FRG is an important tool for addressing nonperturbative problems within quantum field theory. The starting point is the infrared (IR) regularized effective average action $\Gamma_{k}$ [14, 27, 28, 29]. This is a generalization of the standard effective action, where one-particle modes with a momentum less than a scale parameter $k$ are suppressed by means of a regulator function.

The FRG equation for the effective action reads [14, 27]

$$
\begin{aligned}
\partial_{k} \Gamma_{k}[\Phi, \psi] & =\frac{1}{2} \operatorname{Tr}\left\{\partial_{k} R_{k B}\left(\Gamma_{k}^{(2)}[\Phi, \psi]+R_{k B}\right)^{-1}\right\} \\
- & \operatorname{Tr}\left\{\partial_{k} R_{k F}\left(\Gamma_{k}^{(2)}[\Phi, \psi]+R_{k F}\right)^{-1}\right\}
\end{aligned}
$$

where $\Gamma_{k}^{(2)}$ denotes the second functional derivative of $\Gamma_{k}[\Phi, \psi]$ with respect to the field variables and corresponds to the inverse two-point function at the scale $k$. The trace in Eq. (3) denotes a momentum integration and a summation over all internal indices like e.g. flavor, color, and/or Dirac indices. The fields $\Phi$ and $\psi$ denote bosonic and fermionic fields, respectively.

The effective average action $\Gamma_{k}$ governs the dynamics of a theory at the momentum scale $k$ and interpolates between the bare action $\Gamma_{k=\Lambda} \equiv S$ and the full quantum effective action $\Gamma_{k=0}=\Gamma$. The regulator function $R_{k}$, which suppresses the small momentum modes, is to some extent arbitrary. The derivative of this function $\partial_{t} R_{k}$ is peaked at the scale $k$ and thus implements the Wilsonian 
idea of successively integrating out shells in momentum space.

The effect of fluctuations is thus gradually included in $\Gamma_{k}$ by solving the FRG flow equation. In Euclidean space-time

$$
\begin{aligned}
\Gamma_{k}= & \int d^{4} x\left\{\frac{1}{2} Z_{\phi, k}\left(\partial_{\mu} \phi\right)^{2}+Z_{\psi, k} \bar{\psi} \not \partial \psi\right. \\
& \left.+g \bar{\psi}(\sigma+i \vec{\tau} \cdot \vec{\pi} \bar{\gamma}) \psi+U_{k}(\rho, \sigma)\right\},
\end{aligned}
$$

where the field $\rho$ is given by

$$
\rho=\frac{1}{2} \phi^{2}=\frac{1}{2}\left(\sigma^{2}+\vec{\pi}^{2}\right)
$$

and the fermionic field $\psi$ carries two flavors corresponding to the up and down quarks. The matrix $\bar{\gamma}$ is the Euclidean analogue of $\gamma_{5}$.

In the following we assume that each spectral function is dominated by a pole, corresponding to a quasiparticle. Furthermore, we neglect the wavefunction renormalization for both the bosonic and fermionic fields $\left(Z_{\phi, k}=Z_{\psi, k}=1\right)$, i.e. the anomalous dimension is set to zero. Thus, changes of quasiparticle properties in the medium are accounted for, but the fragmentation of singel-particle strength is ignored. Finally, we neglect the scale dependence of the Yukawa coupling $g$ in Eq. (4). Consequently, the only scale dependence we are left with is that of the potential $U_{k}(\rho)$.

This approximation to the average effective action $\Gamma_{k}$ in (4) corresponds to the leading order derivative expansion, which is obtained as the leading term in a systematic expansion in powers of derivatives of the fields. For uniform field configurations, the average effective action $\Gamma_{k}$ evaluated in this approximation is proportional to the effective potential $U_{k}(\rho, \sigma)$, since

$$
\Gamma_{k}=\int d^{4} x U_{k}(\rho, \sigma)
$$

The leading order derivative expansion offers a relatively transparent framework, which nonetheless yields a very useful description of the critical fluctuations near a phase transition.

In addition to defining the form of the effective action, one also needs to specify the regulator functions $R_{k}$. If suitably chosen, the momentum integration is both infrared and ultraviolet finite, leading to a numerically stable solution of the flow equations. We use the so called optimized regulator functions [30],

$$
R_{B, k}^{\mathrm{opt}}\left(q^{2}\right)=\left(k^{2}-q^{2}\right) \theta\left(k^{2}-q^{2}\right),
$$

for bosons and

$$
R_{F, k}^{\mathrm{opt}}(q)=\not\left(\sqrt{\frac{k^{2}}{q^{2}}}-1\right) \theta\left(k^{2}-q^{2}\right)
$$

for fermions. Such regulator functions in general lead to a stabilization of the RG flow and an improved convergence towards the physical theory [30]. Due to the theta function, the regulator acts as a momentum-dependent mass term for all modes with $q^{2} \leq k^{2}$.

\section{A. Flow at finite temperature and chemical potential}

In the previous section we have introduced the basic concepts of FRG. In the following we will use this method to find the nonperturbative thermodynamic potential at finite temperature and density.

We treat bosons and fermions in thermal equilibrium in the standard imaginary time formalism. In thermal equilibrium the (fermion) boson fields satisfy (anti-) periodic boundary conditions in the Euclidean time direction with periodicity $1 / T$. The momentum integration is replaced by a Matsubara sum as follows:

$$
\int \frac{d^{d} q}{(2 \pi)^{d}} \rightarrow T \sum_{n \in \mathbb{Z}} \int \frac{d^{d-1} q}{(2 \pi)^{d-1}}
$$

where

$$
q_{0}(n)=2 n \pi T, \quad q_{0}(n)=(2 n+1) \pi T
$$

for bosons and fermions, respectively.

We employ a modified form of the optimized regulator, where the Euclidean 4-momentum squared $q^{2}$ in Eqs. (7) and (8) is replaced by the 3 -momentum squared $\mathbf{q}^{2}$. Thus, for bosons we use 31]

$$
R_{B, k}^{\mathrm{opt}}(\mathbf{q})=\left(k^{2}-\mathbf{q}^{2}\right) \theta\left(k^{2}-\mathbf{q}^{2}\right)
$$

The finite quark chemical potential $\mu$ is introduced by the following substitution of the time derivative

$$
\partial_{0} \rightarrow \partial_{0}+i \mu
$$

in the fermionic part of the effective action (44). At finite $\mu$, the fermionic regulator function is then of the form

$R_{F, k}^{\mathrm{opt}}(q)=\left(\not q+i \mu \gamma^{0}\right)\left(\sqrt{\frac{\left(q_{0}+i \mu\right)^{2}+k^{2}}{\left(q_{0}+i \mu\right)^{2}+\mathbf{q}^{2}}}-1\right) \theta\left(k^{2}-\mathbf{q}^{2}\right)$.

Clearly, the modified regulators are invariant only under spatial rotations of the momentum $\mathbf{q}$ in a particular frame, but not under Euclidean rotations involving the imaginary time direction. However, this is not a crucial issue in calculations at finite $T$ and $\mu$, where the heat bath defines a preferred frame. Furthermore, the Euclidean invariant form leads to various difficulties, as pointed out in 31]. A great advantage of the regulators (11) and (13) is that, in the quasiparticle approximation, the Matsubara sums for the one-loop diagrams are identical to those appearing in a free theory.

Thus, with the regulators (11) and (13) one obtains the following flow equation for the thermodynamic potential density $(\Omega=(T / V) \Gamma)$ 

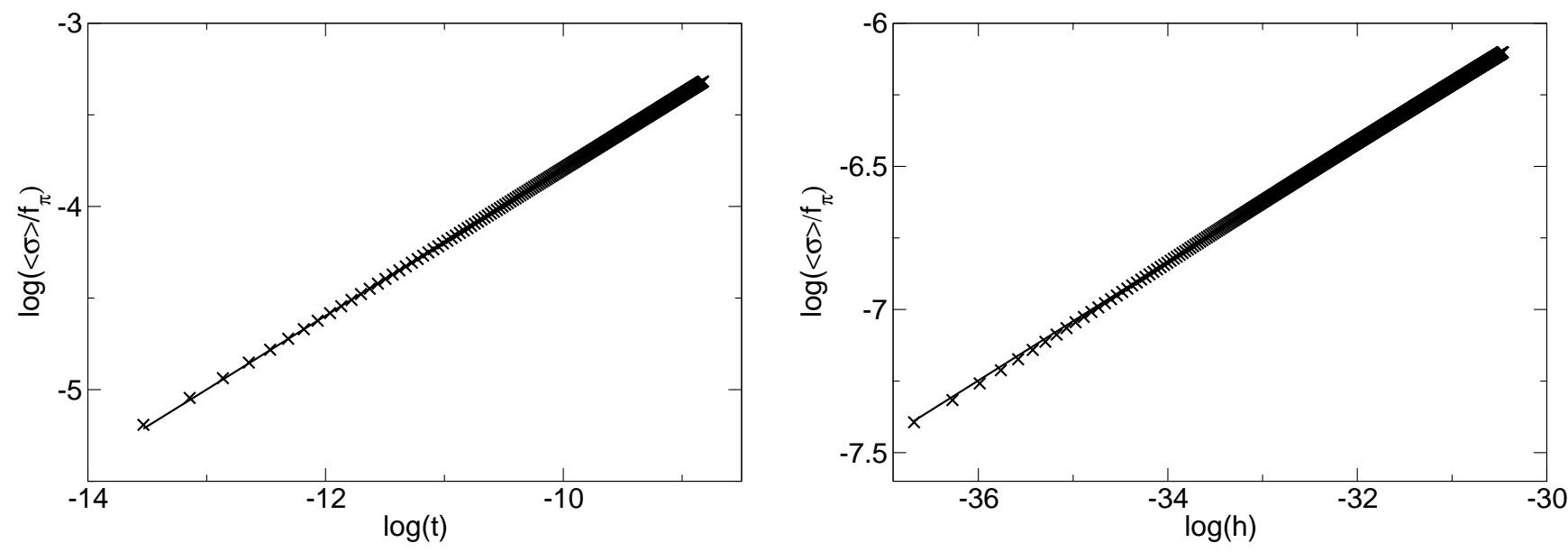

FIG. 2: Scaling of the order parameter with $t$ and $h$. The logarithmic fits yield the critical exponents $\beta$ (left panel) and $\delta$ (right panel).

$$
\partial_{k} \Omega_{k}(T, \mu)=\frac{k^{4}}{12 \pi^{2}}\left[\frac{3}{E_{\pi}}\left(1+2 n_{B}\left(E_{\pi}\right)\right)+\frac{1}{E_{\sigma}}\left(1+2 n_{B}\left(E_{\sigma}\right)\right)-\frac{2 \nu_{q}}{E_{q}}\left(1-n_{F}\left(E_{q}\right)-\bar{n}_{F}\left(E_{q}\right)\right)\right]
$$

where $n_{B}\left(E_{\pi, \sigma}\right)$ and $n_{F}\left(E_{q}\right), \bar{n}_{F}\left(E_{q}\right)$ are the bosonic and fermionic distribution functions

$$
\begin{gathered}
n_{B}\left(E_{\pi, \sigma}\right)=\frac{1}{e^{E_{\pi, \sigma} / T}-1} \\
n_{F}\left(E_{q}\right)=\frac{1}{e^{\left(E_{q}-\mu\right) / T}+1}, \quad \bar{n}_{F}\left(E_{q}\right)=\frac{1}{e^{\left(E_{q}+\mu\right) / T}+1} .
\end{gathered}
$$

Here

$$
E_{\pi}=\sqrt{k^{2}+\bar{\Omega}_{k}^{\prime}}, E_{\sigma}=\sqrt{k^{2}+\bar{\Omega}_{k}^{\prime}+2 \rho \bar{\Omega}_{k}^{\prime \prime}}
$$

is the pion and sigma energies, where $\bar{\Omega}_{k}^{\prime}$ and $\bar{\Omega}_{k}^{\prime \prime}$ denote the first and the second derivatives of the thermodynamic potential with the explicit symmetry breaking term removed, $\bar{\Omega}_{k}=\Omega_{k}+c \sqrt{2 \rho_{k}}$, with respect to $\rho$ and

$$
E_{q}=\sqrt{k^{2}+2 g^{2} \rho}
$$

the quark energy. Finally, the quark degeneracy is given by $\nu_{q}=2 N_{c} N_{f}$.

The flow equation (14) is identical to the one obtained within the proper time renormalization group (PTRG) scheme [9, 18]. In fact, it was shown by Litim [30] that, in vacuum, the PTRG scheme, with a properly chosen cutoff function, is equivalent to the optimized FRG flow in the leading order derivative expansion.

The flow equation for $\Omega_{k}(T, \mu)$ can be solved numerically by either discretizing the potential on a grid [9] or expanding it in powers of the fields $(\sigma, \rho)$. In this paper, we employ the second method and expand the potential $\Omega_{k}(T, \mu)$ in a Taylor series around the minimum at $\sigma_{0}=\sqrt{2 \rho_{0}}$

$$
\bar{\Omega}_{k}(T)=\sum_{m} \frac{a_{m, k}(T)}{m !}\left(\rho_{k}-\rho_{0}\right)^{m} .
$$

The symmetry breaking term is treated explicitly, leading to

$$
\Omega_{k}(T)=\bar{\Omega}_{k}(T)-c \sigma_{k}
$$

The stationarity condition

$$
\left.\frac{\partial \Omega_{k}(T)}{\partial \sigma}\right|_{\min }=0
$$

determines the position of the physical minimum at the scale $k$. Thus, for $c \neq 0$, i.e., for an explicitly broken symmetry, we find

$$
c=a_{1} \sigma_{0}
$$

which relates the coupling $a_{1}$ and the expectation value of the scalar field $\sigma_{0}$.

We truncate the expansion in Eq. (15) at $m=3$. Using the identity 45.

$$
\frac{d \Omega_{k}^{\prime}}{d k}=\frac{\partial \Omega_{k}^{\prime}}{\partial \rho} \frac{d \rho}{d k}+\frac{\partial \Omega_{k}^{\prime}}{\partial k}
$$

and the corresponding relations for $\Omega_{k}^{\prime \prime}$ and $\Omega_{k}^{\prime \prime \prime}$, we then 
obtain the flow equations

$$
\begin{aligned}
d_{k} a_{0} & =\frac{c}{\sqrt{2 \rho_{k}}} d_{k} \rho_{k}+\partial_{k} \Omega_{k}, \\
d_{k} \rho_{k} & =-\frac{1}{\left(c /\left(2 \rho_{k}\right)^{3 / 2}+a_{2}\right)} \partial_{k} \Omega_{k}^{\prime}, \\
d_{k} a_{2} & =a_{3} d_{k} \rho_{k}+\partial_{k} \Omega_{k}^{\prime \prime}, \\
d_{k} a_{3} & =\partial_{k} \Omega_{k}^{\prime \prime \prime},
\end{aligned}
$$

where we have introduced the shorthand notation $d_{k}=$ $d / d k$. In Eq. (19) the terms $\partial \Omega_{k}^{\prime} / \partial \rho$ etc. are evaluated using the expansion (15) truncated at $m=3$.

In the following we consider only the case of vanishing net baryon density, i.e., $\mu=0$, while the external field $c$ is kept finite. Results for the chiral limit are then obtained by letting $c \rightarrow 0$.

The flow equations are solved numerically starting at a cutoff scale $\Lambda=1.2 \mathrm{GeV}$. The initial values for the parameters are chosen such that the physical pion mass $m_{\pi}=138 \mathrm{MeV}$, the pion decay constant $f_{\pi}=93 \mathrm{MeV}$ and the sigma mass $m_{\sigma}=600 \mathrm{MeV}$ are reproduced in vacuum, using the regulators (11) and (13), for a constituent quark mass of $m_{q}=300 \mathrm{MeV}$. The strength of the external field $c_{0}$ and the Yukawa coupling $(g=3.2)$ are fixed by the pion mass and the constituent quark mass. The pion and the sigma masses, at a given momentum scale $k$, are given by

$$
m_{\pi, k}^{2}=\frac{c}{\sqrt{2 \rho_{k}}}, \quad m_{\sigma, k}^{2}=\frac{c}{\sqrt{2 \rho_{k}}}+2 \rho_{k} a_{2, k},
$$

while the constituent quark mass is obtained from

$$
m_{q, k}^{2}=2 g^{2} \rho_{k} .
$$

The numerical solution of the flow equations yields a non-perturbative thermodynamic potential at finite temperatures. This can then be used to explore the critical properties of thermodynamical observables in the vicinity of the chiral phase transition.

\section{CRITICAL BEHAVIOR AND $O(4)$ SCALING}

It is well known that the effect of critical fluctuations on the properties of a system close to a second-order phase transition can be efficiently computed by means of the Wilsonian renormalization group [32]. A systematic account of fluctuations is essential for a realistic description of the critical properties of such systems. The FRG approach offers a powerful tool for computing not only universal quantities, like the critical exponents, but also the full thermodynamics of a strongly interacting manybody systems. Here we focus on the critical exponents of the chiral phase transition.

In the chiral limit, the chiral quark-meson model exhibits a second order phase transition. The critical behavior of this model (and of QCD) is expected to be governed by the fixed point of the $O(4)$ Heisenberg model in three dimensions. Universality class arguments lead to predictions for the functional form of various thermodynamic quantities in the vicinity of the critical temperature $T_{c}$, the critical exponents. These all emerge from the scaling form of the singular part of the free energy density

$$
\mathcal{F}_{s}(t, h)=b^{-d} \mathcal{F}_{s}\left(b^{y_{t}} t, b^{y_{h}} h\right) .
$$

The scaling relation (23) determines the properties of $\mathcal{F}_{s}$ under a scale transformation of all lengths by a factor $b$. There are two relevant parameters in $\mathcal{F}_{s}(t, h)$ which control the critical behavior: the reduced temperature $t=\left(T-T_{c}\right) / T_{c}$ and the reduced external field $h=c / c_{0}$. The dimensionless quantity $\mathcal{F}_{s}$ is the free energy density in units of $f_{\pi}^{2} m_{\pi}^{2}$. All physical information concerning the phase transition can be encoded in the equation of state which relates the order parameter, the external field and the reduced temperature in the vicinity of the critical point. The leading singularities at the critical point are then completely controlled by the thermal and the magnetic critical exponents, $y_{t}$ and $y_{h}$.

In this section we compute critical properties and the equation of state of the quark meson model using the FRG method and confront our results with those of the $O(4)$ universality class. We compare the behavior of various physical observables in the critical region with that expected from the universal scaling function (23) and extract their critical exponents.

\section{A. Scaling of the order parameter}

A phase transition involving spontaneous symmetry breaking, is signalled by the vanishing of an order parameter, when approached from the broken symmetry phase. In the quark meson model, a possible order parameter related to chiral symmetry breaking is obtained by differentiating the free energy with respect to the external field $c$. Thus, it follows from Eqs. (2) and (5) that the order parameter can be identified with the thermodynamic average of the sigma field

$$
\langle\sigma\rangle=\sigma_{0}=\sqrt{2 \rho_{0}} .
$$

In the FRG approach, the value of the order parameter depends on the scale $k$; the flow of $\langle\sigma\rangle$ follows from equation Eq. (20b). The physical value of the order parameter at a given temperature $T$ is then given by $\sqrt{2 \rho_{k \rightarrow 0}}$, where $\rho_{k \rightarrow 0}$ is the value obtained at the end of the flow $(k \rightarrow 0)$.

In Fig. 1 we show the resulting dependence of the order parameter on the reduced temperature $t$ for several values of the external field $h$. In the chiral limit, i.e. for $h=0$, the order parameter $\langle\sigma\rangle$ smoothly decreases with increasing $t$ and finally vanishes at the critical temperature $t=0$. Such a behavior is characteristic of a system with a second order phase transition. For finite $h$, the chiral symmetry of the Lagrangian is explicitly broken and 

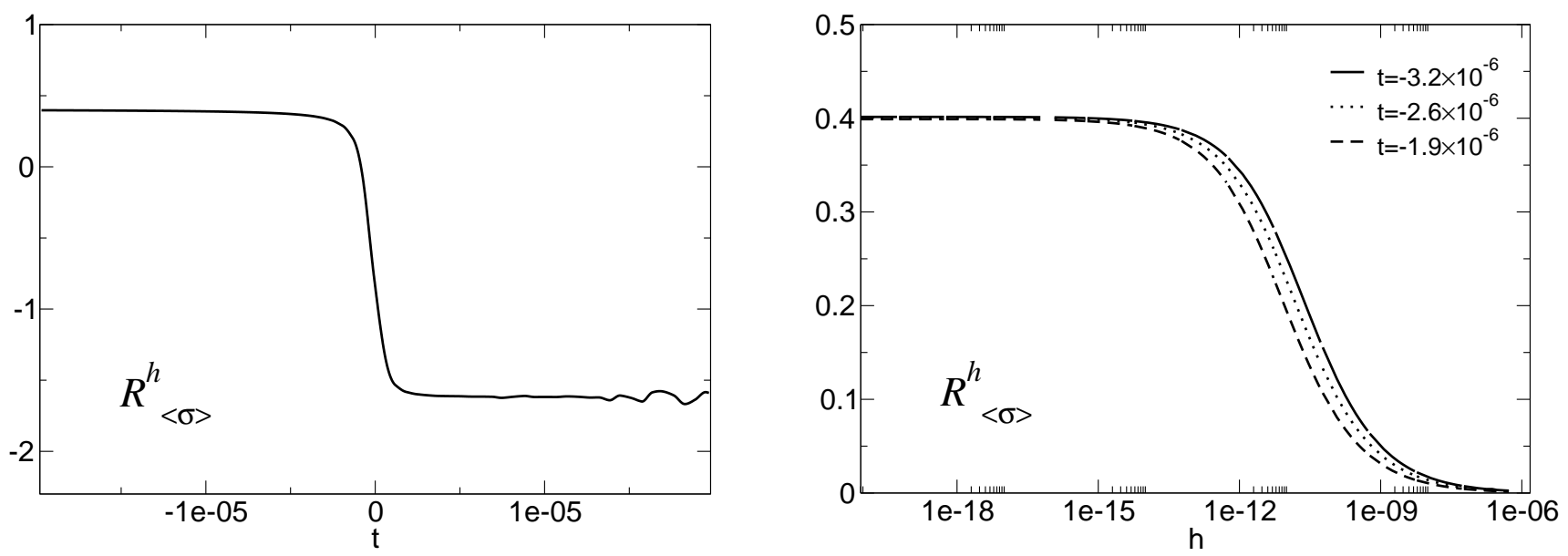

FIG. 3: The ratio $R_{\langle\sigma\rangle}^{h}$ as a function of $t$ (left), for $h=6 \times 10^{-13}$, and as a function of $h$ for three values of the reduced temperature $t$.

the second order phase transition turns into a crossover transition. Thus, for small but finite $h$, the order parameter $\langle\sigma\rangle$ changes fast in a narrow temperature interval, at the so called pseudo-critical temperature. However, in this case the order parameter remains non-zero, even at very high temperatures, as seen in Fig. 1 and there is no true phase transition.

In the vicinity of the critical point the scaling of the order parameter $\langle\sigma\rangle$ can be obtained from the universal form of the free energy. Indeed, by choosing the scale factor $b$ in the Eq. (23) such that $b^{y_{h}} h=1$ or $b^{y_{t}}|t|=1$ one finds

$$
\mathcal{F}_{s}=h^{d \nu_{c}} \mathcal{F}_{s}\left(t h^{-1 / \beta \delta}, 1\right)
$$

and

$$
\mathcal{F}_{s}=|t|^{d \nu} \mathcal{F}_{s}\left(t|t|^{-1}, h|t|^{-\beta \delta}\right),
$$

respectively. Consequently, using

$$
\langle\tilde{\sigma}\rangle=-\frac{\partial \mathcal{F}_{s}}{\partial h},
$$

where $\langle\tilde{\sigma}\rangle=\langle\sigma\rangle / f_{\pi}$ is the reduced order parameter, one finds the corresponding equation of state for the magnetization

$$
\langle\tilde{\sigma}\rangle=h^{1 / \delta} F_{h}(z)
$$

and

$$
\langle\tilde{\sigma}\rangle=|t|^{\beta} \mathcal{F}_{s}^{\prime}\left(t|t|^{-1}, h|t|^{-\beta \delta}\right),
$$

with $z \equiv t h^{-1 / \beta \delta}$ and the universal function $F_{h}(z)$ is obtained from Eq. (25) by differentiation with respect to $h$. The prime on $\mathcal{F}_{s}$ in (29) denotes the derivative with respect to the second argument.

The well known scaling behavior of the magnetization

$$
\langle\tilde{\sigma}\rangle=\left\{\begin{array}{ll}
B(-t)^{\beta}, & h=0, t<0 \\
B_{c} h^{1 / \delta}, & t=0, h>0
\end{array},\right.
$$

follows from Eqs. (28) and (29). The constants $B_{c}=$ $F_{h}(0)$ and $B=\mathcal{F}_{s}^{\prime}(-1,0)$ characterize the magnetization on the so called coexistence line [33] $(t<0, h=0)$ and at the pseudo-critical point $(t=0, h>0)$. In the derivation of Eqs. (25) - (30) we have used the definitions of the thermal and magnetic critical exponents

$$
y_{t}=\frac{1}{\nu}, \quad y_{h}=\frac{1}{\nu_{c}}=\frac{\beta \delta}{\nu},
$$

as well as the scaling relations

$$
\delta-1=\frac{\gamma}{\beta}, \quad \gamma=\nu(2-\eta), \quad d \nu=\beta(1+\delta)
$$

and

$$
\alpha=2-d \nu
$$

In the chiral limit, the scaling behavior of the order parameter is controlled by the critical exponent $\beta$, when the critical temperature $t=0$ is approached from below. Thus, $\beta$ is given by the slope in a double-logarithmic plot of $\langle\tilde{\sigma}\rangle$ versus $t$. The critical exponent $\delta$, on the other hand, characterizes the scaling of $\langle\tilde{\sigma}\rangle$ as $h \rightarrow 0$ for $t=0$.

In Fig. 2 we show the scaling properties of $\langle\tilde{\sigma}\rangle$ close to the critical point $(t \rightarrow 0)$ for vanishing external field as well as the scaling with $h$ at the (pseudo)critical point $(t=0)$. In both cases the order parameter shows the expected scaling behavior. This demonstrates that, in the parameter range considered, the free energy of the quark meson model is dominated by the singular part Eq. (23). The critical exponents extracted from a fit to the results of Fig. 2 are

$$
\beta \simeq 0.402 \quad \text { and } \quad \delta \simeq 4.818 .
$$

Both values are consistent with $O(4)$ universality. Indeed, in a Monte Carlo simulation of the threedimensional $O(4)$ spin model, Kanaya and Kaya 26] 
find $\beta=0.3836(46)$ and $\delta=4.851(22)$. The agreement with this presicion calculation is, given our simple Ansatz, quite satisfactory. Consequently, we conclude that the critical properties of the chiral order parameter in the quark-meson model are indeed governed by the $O(4)$ universality class. Our results, obtained in the relatively simple leading order derivative expansion, illustrate the efficiency of the FRG approach to correctly account for a non-perturbative, long distance physics near a phase transition.

In the mean field approximation one also finds scaling of the order parameter $\langle\tilde{\sigma}\rangle$ close to the critical point, but with different critical exponents, $\beta=0.5$ and $\delta=$ 3. Thus, the singularity at the chiral phase transition is substantially modified by fluctuations.

In the formulation of the flow equation for the quarkmeson model we have neglected the wavefunction renormalization for both bosonic and fermionic fields. The good agreement with the $O(4)$ lattice results as well as with previous FRG studies [19], where the anomalous dimension was taken into account, indicates that the influence of the wavefunction renormalization on the critical properties at the chiral phase transition is quite small.

So far we have discussed the scaling of the order parameter $\langle\tilde{\sigma}\rangle$ with $t$ for $h=0$ in the chirally broken phase and with $h$ at $t=0$. In the chiral limit, the order parameter vanishes identically in the symmetric phase, i.e. for $t>0$. Thus, the order parameter does not exhibit a singularity when the critical point is approached from above. However, since for $h \neq 0$ the order parameter is always non-zero, it is interesting to explore its behavior for small but finite values of $h$, when the critical point is approached from the broken $(t<0)$ as well as from the symmetric $(t>0)$ phase. The scaling behavior of the order parameter is in this case characterized by a socalled effective critical exponent [24]. For a given $h$, this exponent is defined by

$$
R_{\langle\sigma\rangle}^{h}:=\frac{d \log \langle\tilde{\sigma}\rangle}{d \log t}=\frac{t}{\langle\tilde{\sigma}\rangle} \frac{\partial\langle\tilde{\sigma}\rangle}{\partial t} .
$$

From Eqs. (30) and (35) it is clear that for $h=0$ and $t<0$, the ratio $R_{\langle\sigma\rangle}^{h=0}$ coincides with the critical exponent $\beta$. For $h \neq 0$, Eq. (35) can be used to extract the leading singularity of the order parameter in the symmetric region close to the critical point.

In the left panel of Fig. 3 we show the resulting dependence of the effective critical exponent (35) on the reduced temperature for a fixed, very small, value of the external field $h$. The exponent $R_{\langle\sigma\rangle}^{h}$ exhibits the expected scaling behavior. In the broken $(t<0)$ as well as in the symmetric $(t>0)$ phase, it is essentially independent of $t$. The effective critical exponents are different in the broken and symmetric phases. A weak external field $h \simeq 0$ and $t<0$ corresponds to $z \rightarrow-\infty$ in Eq. (28). It follows that the asymptotic form of the scaling function is given by [34] $F_{h}(z) \sim(-z)^{\beta}$ for $z \rightarrow-\infty$. Thus, in the broken phase $(t<0)$ the exponent $R_{\langle\sigma\rangle}^{h}$ is, as expected, consis- tent with the value of the critical exponent $\beta$, extracted from the scaling properties (30) of the order parameter.

In the right panel of Fig. 3. we show the dependence of the effective critical exponent upon $h$ in the broken phase for three different values of $t$. For $t<0$, the limit $h \rightarrow 0$ corresponds to $z \rightarrow-\infty$ and consequently $R_{\langle\sigma\rangle}^{h}$ again converges to the critical exponent $\beta$. On the other hand, the limit $h \rightarrow \infty$ corresponds to $z \rightarrow 0$. In this case, the order parameter $\langle\tilde{\sigma}\rangle \sim h^{1 / \delta}$ is, to leading order, independent of temperature and the effective critical exponent approaches zero. The transition between the two regimes takes place at $|z| \sim 1$.

Also in the symmetric phase $(t>0)$, the exponent $R_{\langle\sigma\rangle}^{h}$ shows a power law behavior, however with a different value of the exponent. In the transition region, $R_{\langle\sigma\rangle}^{h}$ behaves like a smoothened step function, interpolating between the asymptotic regions. The transition region extends from $z \sim-1$ to $z \sim 1$. Hence the width of the step in $t$ is expected to scale with $h^{1 / \beta \delta}$. The effective critical exponent changes from $R_{\langle\sigma\rangle}^{h} \simeq 0.4$ in the broken phase to $R_{\langle\sigma\rangle}^{h} \simeq-1.5$ in the symmetric phase. This change in the critical behavior of the order parameter can be understood using the universal scaling behavior of the singular part of the free energy. To see this, we first write the equation of state (28) and (29) in the WidomGriffiths form

$$
y=f(x)
$$

where

$$
y \equiv \frac{h}{\langle\tilde{\sigma}\rangle^{\delta}} \quad, \quad x \equiv \frac{t}{\langle\tilde{\sigma}\rangle^{1 / \beta}} .
$$

The scaling relations (30) are, modulo constant factors, obtained if $f(0)=1$ and $f(-1)=0$. The constant factors are recovered by a simple rescaling of $t$ and $h$. A comparison of the Widom-Griffiths form of the equation of state (36) with that given in Eq. (28), shows that the following relations hold:

$$
F_{h}(z)=f(x)^{-1 / \delta}, \quad z=x y^{-1 / \beta \delta} .
$$

Consequently, Eq. (28) can be rewritten as

$$
\langle\tilde{\sigma}\rangle=B_{c} h^{1 / \delta} f(x)^{-1 / \delta},
$$

where we have reinstated the constant factor $B_{c}$. This equation of state can be used to explore the scaling properties of $\langle\tilde{\sigma}\rangle$ when approaching the critical point from the symmetric phase. For $t>0$ and for $h \rightarrow 0$, the chiral order parameter (normalized to its value in vacuum) is very small. Consequently, the argument $x$ of the function $f(x)$ in equation (39) is very large and the scaling behavior of $\langle\tilde{\sigma}\rangle$ for $t>0$ and for small but finite $h$ is determined by the asymptotic form of the scaling function $f(x)$ for $x \rightarrow \infty$. In this limit, $f(x)$ is given by Griffiths' analyticity condition 35],

$$
f(x)=\sum_{n=1}^{\infty} f_{n} x^{\gamma-2(n-1) \beta} .
$$




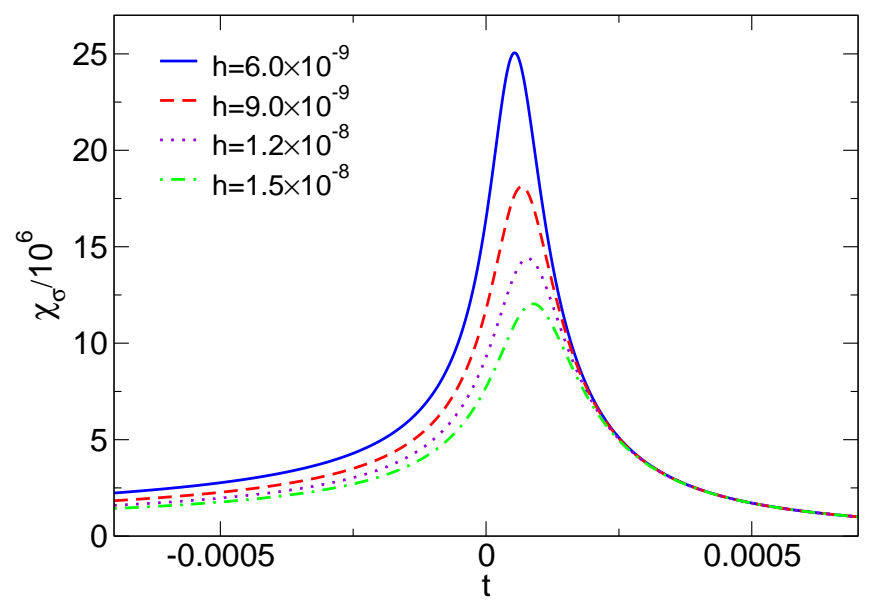

FIG. 4: The susceptibility $\chi_{\sigma}$ as a function of the reduced temperature $t$ for several values of the field $h$.

Retaining only the leading term, $f(x) \simeq x^{\gamma}$, one finds that for $t>0$ and $h \rightarrow 0$ the equation of state scales as

$$
\langle\tilde{\sigma}\rangle \sim t^{-\gamma} h .
$$

Thus, for $t>0$ and $h \neq 0$, we expect $R_{\langle\sigma\rangle}^{h}=-\gamma$, in $\operatorname{good}$ agreement with the results shown in Fig. 3. This result implies [34] that for $z \rightarrow \infty, F_{h}(z) \simeq z^{-\gamma}$. We note that in the chiral limit $(h=0)$, the order parameter vanishes identically for $t>0$, as expected.

From the scaling behavior of $\langle\tilde{\sigma}\rangle$ shown in the left panel of Fig. 3, we find $\gamma \simeq 1.6$. This is consistent with the lattice result for the $O(4)$ spin system obtained in ref. [26], $\gamma \simeq 1.5$. Thus, the FRG method applied to the quark meson model yields a scaling behavior of the order parameter, which is consistent with that found for the $O(4)$ universality class. This is the case at the critical point, on the coexistence line and when approaching the critical point from the high temperature phase for a finite value of the external magnetic field.

\section{B. Scaling of the chiral susceptibilities}

The critical properties of a thermodynamic system can be explored by studying the fluctuations of various observables. In particular, the fluctuations of the order parameter probe the order of the phase transition and the position of a possible critical end point. In statistical physics, fluctuations are reflected in the corresponding susceptibilities $\chi$. In the case of the chiral order parameter, the corresponding susceptibility is defined as the response to a change of the external field $h$; the susceptibility $\chi$ is obtained by taking the second derivative of the effective potential with respect to $h$. The susceptibility in other channels is computed analogously. It follows that the susceptibilities are inversely proportional to the corresponding mass squared. Consequently, the divergence of a susceptibility, e.g. at the critical end point, signals a zero-mass mode of the corresponding effective field.

In the chiral quark-meson model, we are not only dealing with fluctuations of the order parameter, i.e. of the sigma field, but also with a pseudo-Goldstone mode, corresponding to fluctuations of the pion field. The sigma and the pion represent the longitudinal and transverse modes of the $O(4)$ field $\phi$, respectively. One therefore distinguishes between the longitudinal $(\sigma)$ and transverse $(\pi)$ order parameter susceptibilities.

The longitudinal susceptibility is given by the derivative of the order parameter with respect to the external field

$$
\chi_{l}=\chi_{\sigma}=\frac{\partial\langle\tilde{\sigma}\rangle}{\partial h},
$$

whereas the transverse susceptibility, which is directly related to the fluctuation of the Goldstone modes, is obtained from

$$
\chi_{t}=\chi_{\pi}=\frac{\langle\tilde{\sigma}\rangle}{h} .
$$

The latter expression is a direct consequence of the $O(4)$ invariance of the free energy for $h=0$ and follows from the corresponding Ward identity [36].

The critical behavior of the longitudinal and transverse chiral susceptibility can be obtained from the scaling form of the singular part of the free energy or directly from the magnetic equation of state (39).

In Fig. 4 we show the longitudinal susceptibility $\chi_{\sigma}$ as a function of the reduced temperature for several values of the symmetry breaking field $h$. At finite $h$, this susceptibility shows a peak structure with a maximum at the pseudo-critical temperature $t_{\max }(h)$. With decreasing $h$, there is a shift of $t_{\max }$ and an increase of the fluctuations at the pseudo-critical point; in the chiral limit $\chi_{\sigma}$ diverges at the transition temperature, because the mass of the sigma field vanishes.

Using the results shown in the Fig. (4) one can define a pseudo-critical line in the $(t, h)$-plane that characterizes the dependence of the transition temperature $t_{\max }$ on the symmetry breaking field $h$. Renormalization group arguments imply that the pseudo-critical temperature and the maximum of the longitudinal susceptibility should exhibit universal scaling according to [37]:

$$
t_{\max }(h) \simeq T_{p} h^{1 /(\gamma+\beta)}, \quad \chi_{\sigma}\left(t_{\max }, h\right) \simeq C_{p} t_{\max }^{-\gamma},
$$

with $O(4)$ critical exponents.

In Fig. 5 we confront the scaling behavior of the susceptibility with Eq. (44). The critical exponents are extracted by performing a linear logarithmic fit to the pseudo-critical temperature and the maximum value of the susceptibility. We find $1 /(\gamma+\beta)=0.494$ and $\gamma=1.618$, which implies $\beta=0.406$, in good agreement with the results obtained above using Eqs. (30) and (411). Thus, the scaling of of $\chi_{\sigma}$ with the symmetry breaking field $h$ is also consistent with $O(4)$ universality. 

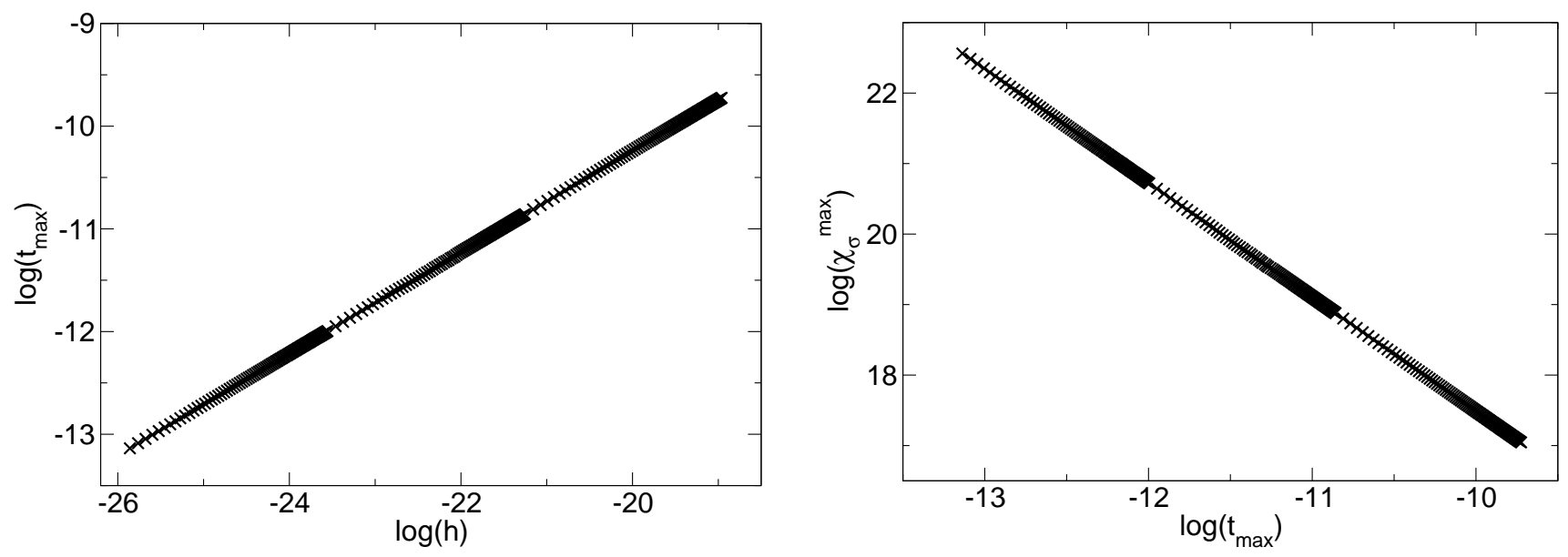

FIG. 5: Scaling of the pseudo-critical temperature defined by the maximum of the susceptibility $\chi_{\sigma}$ (left) and of the maximum value of $\chi_{\sigma}$ (right).

In the symmetric phase, the longitudinal and the transversal susceptibilities coincide $\left(\chi_{\sigma}=\chi_{\pi}\right)$. Consequently, the strength of the singularities at the critical point $t=0$ are controlled by the same critical exponents.

To find the scaling behavior of $\chi$ when approaching the critical point from the symmetric phase we use the Widom-Griffiths form of the magnetic equation of state (39) together with the large- $x$ expansion of the scaling function $f(x)$.

From Eqs. (41), (42) and (43) it is clear that for $h \rightarrow 0$ in the symmetric phase $(t>0)$

$$
\chi_{\sigma}=\chi_{\pi} \simeq t^{-\gamma} .
$$

Thus, when approaching the critical point from the symmetric phase, the scaling behavior of the longitudinal and

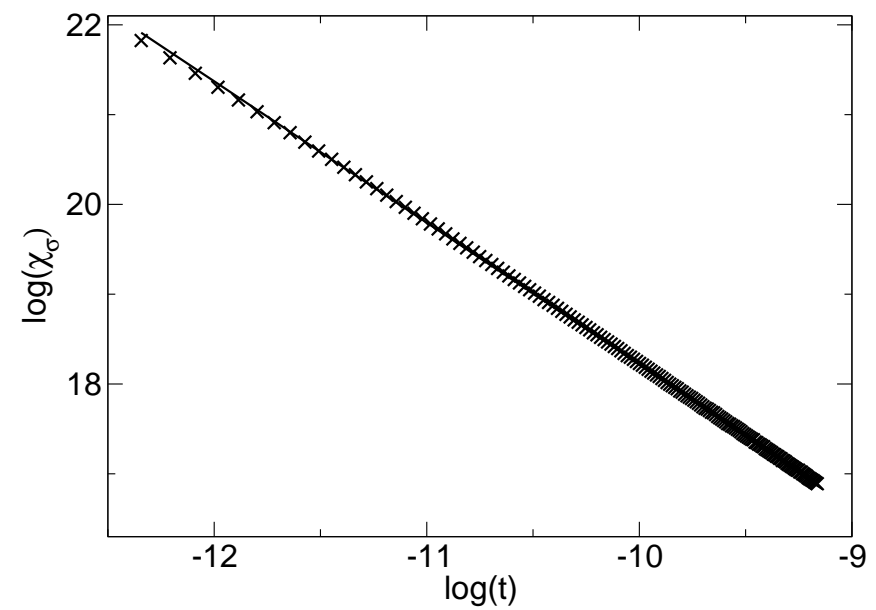

FIG. 6: Double logarithmic plot of the longitudinal susceptibility in the symmetric phase for a small $h$ as a function the reduced temperature. transverse susceptibility is controlled by the critical exponent $\gamma$.

In Fig. [6 we show the scaling behavior of the susceptibility for $t>0$ and in the limit of $h \rightarrow 0$. From the slope of the line we obtain $\gamma=1.575$, in good agreement the value obtained from the analysis of the scaling of the chiral susceptibility $\chi_{\sigma}$ following Eq. (44). The small difference is may be due to the uncertainty in the determination of the critical temperature.

In the broken phase, on the coexistence line, the critical behavior of the longitudinal and transverse susceptibilities differ. In the chiral limit, the order parameter is finite in the broken phase as long as $t<0$. Thus, for $T<T_{c}$ the transverse susceptibility (43) diverges in the chiral limit, $\chi_{\pi} \sim h^{-1}$, due to the appearance of Goldstone bosons. A less obvious result is the divergence of the longitudinal susceptibility on the coexistence line, where to leading order $\chi_{\sigma} \sim h^{-1 / 2}$ [7, 33].

The scaling properties of the susceptibilities can be obtained directly from the Widom-Griffiths form of the magnetic equation of state. Indeed, using Eqs. (39), (42) and (43) one finds

$$
\chi_{\sigma}=B_{c} h^{1 / \delta-1} \frac{\beta f(x)^{1-1 / \delta}}{\beta \delta f(x)-x f^{\prime}(x)},
$$

for the longitudinal and

$$
\chi_{\pi}=B_{c} h^{1 / \delta-1} f(x)^{-1 / \delta},
$$

for the transverse susceptibility.

In the vicinity of the coexistence line, where $x \rightarrow-1$, the scaling function is, in a three-dimensional system, found to be of the form [33, 38]

$$
f(x) \approx c_{f}(1+x)^{2} .
$$

Using (48) in (46) and (47), one then finds the critical behavior of the susceptibilities for $t<0$ and $h \rightarrow 0$ :

$$
\chi_{\sigma} \sim h^{-1 / 2}(-t)^{\beta-(\beta \delta) / 2}
$$



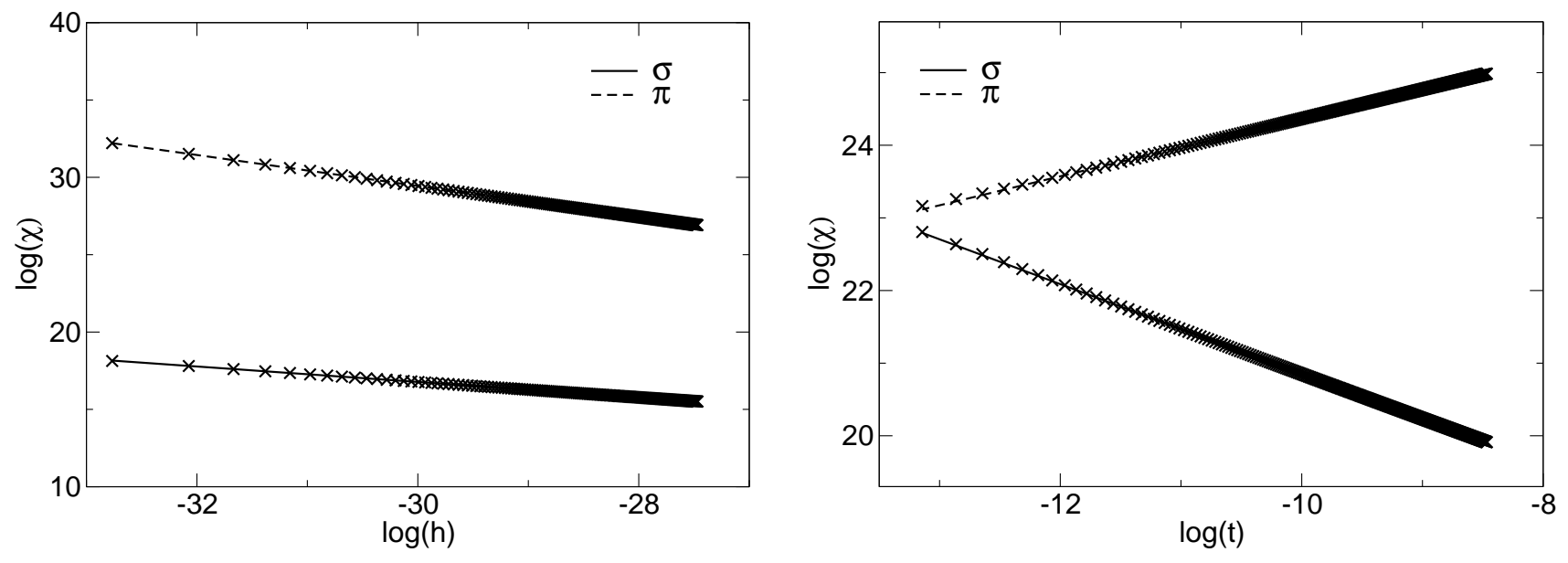

FIG. 7: Scaling of $\chi_{\sigma}$ and $\chi_{\pi}$ in the broken phase $(t<0)$ as functions of $h$ (left) and $t$ (right)

and

$$
\chi_{\pi} \sim h^{-1}(-t)^{\beta}
$$

The divergence of the susceptibilities on the coexistence line is due to the transverse modes [33], whose mass term is proportional to the symmetry-breaking term, $m_{\pi}^{2} \sim$ $h$. The different exponents for $\chi_{\sigma}$ and $\chi_{\pi}$ reflect the direct and indirect dependence of the susceptibilities on the soft transverse modes. The critical behavior of the longitudinal susceptibility close to the coexistence line, fixes the exponent of the subleading term of the scaling function defined in (28) for $z<0$

$$
F_{h}(z) \sim(-z)^{\beta}\left(1+C(-z)^{-\beta \delta / 2}\right),
$$

where $C$ is a constant.

In Fig. 7 we show log-log plots of the longitudinal and transverse susceptibilities, obtained by solving the flow equations for the quark meson model (20), as functions of $h$ and $t$ near the coexistence line. A linear fit to the results shown on the left yields 0.499 and 1.000 for the critical exponents of $\chi_{\sigma}$ and $\chi_{\pi}$, in excellent agreement with the expected scaling behavior with $h$ implied by Eqs. (49) and (50). The corresponding fit to the $t$-dependence, shown in the right plot, gives $(\beta-\beta \delta / 2)=-0.617$ and $\beta=0.395$, which implies $\delta=5.124$, in fair agreement with our previous results for the critical exponents $\beta$ and $\delta$ obtained from the scaling of the order parameter.

In order to exhibit the scaling behavior of the susceptibilities in a broad parameter range, which covers both the broken and symmetric phases, we introduce the ratios

$$
R_{\chi_{\pi, \sigma}}^{h}=\frac{t}{\chi_{\pi, \sigma}} \frac{\partial \chi_{\pi, \sigma}}{\partial t}
$$

for the transverse $\chi_{\pi}$ and the longitudinal $\chi_{\sigma}$ susceptibilities. The ratios $R_{\chi_{\pi, \sigma}}^{h}$ define effective critical exponents, analogous to Eq. (35).
In Fig. 8 we summarize the $t$ and $h$ dependence of the $R_{\chi_{\pi}}^{h}$ and $R_{\chi_{\sigma}}^{h}$ in the vicinity to the critical point. On the left we show how the critical exponents of the susceptibilities interpolate between the broken and symmetric phases, for fixed $h$, while on the right the $h$-dependence of $R_{\chi_{\pi, \sigma}}^{h}$ in the symmetric phase is illustrated for a few values of $t$.

In the symmetric phase, $\chi_{\pi}$ and $\chi_{\sigma}$ coincide and their critical behavior is determined by the same exponent $(\gamma \sim 1.58)$, as seen in Fig. 8-left. This corresponds to the limit $z \rightarrow \infty$, and is consistent with the effective critical exponent obtained for the order parameter for $t>0$. The dependence upon $h$ in the symmetric phase, shown on the right, is also identical for the longitudinal and transverse susceptibilities. Here the $z \rightarrow \infty$ limit corresponds to $h \rightarrow 0$ for fixed $t>0$. Thus, also in this limit, $R_{\chi_{\pi, \sigma}}^{h} \rightarrow-\gamma$, as shown in Fig. 8-right. Again, the $h \rightarrow \infty$ limit corresponds to $z \rightarrow 0$, where the order parameter and consequently also the susceptibility approaches a temperature independent function of $h$ and the corresponding effective critical exponent converges to zero with increasing $h$. The transition between the two regimes again takes place at $z \simeq 1$.

In the broken phase (see Fig. 8-left), the critical exponents differ, $R_{\chi_{\sigma}}^{h} \simeq \beta(1-\delta / 2)$ and $R_{\chi_{\pi}}^{h} \simeq \beta$, in complete agreement with Eqs. (49) and (50).

In section $\Pi$ II $\mathrm{A}$ we determined the critical exponent $\delta$ by studying the scaling of the order parameter $\langle\tilde{\sigma}\rangle$ with $h$ for $t=0$. We note that this critical exponent may be determined in an alternative way, by exploiting the scaling properties of the susceptibilities $[25,39,40] \chi_{\pi}$ and $\chi_{\sigma}$. Consider the ratio

$$
\Delta(t, h)=\frac{\chi_{\pi}^{-1}}{\chi_{\sigma}^{-1}}=\frac{h}{\langle\tilde{\sigma}\rangle} \frac{\partial\langle\tilde{\sigma}\rangle}{\partial h},
$$

which has the useful property that its value at $t=0$ is independent of $h$ within the scaling region. Indeed, using the parametrization of the susceptibilities (46) and (477) 

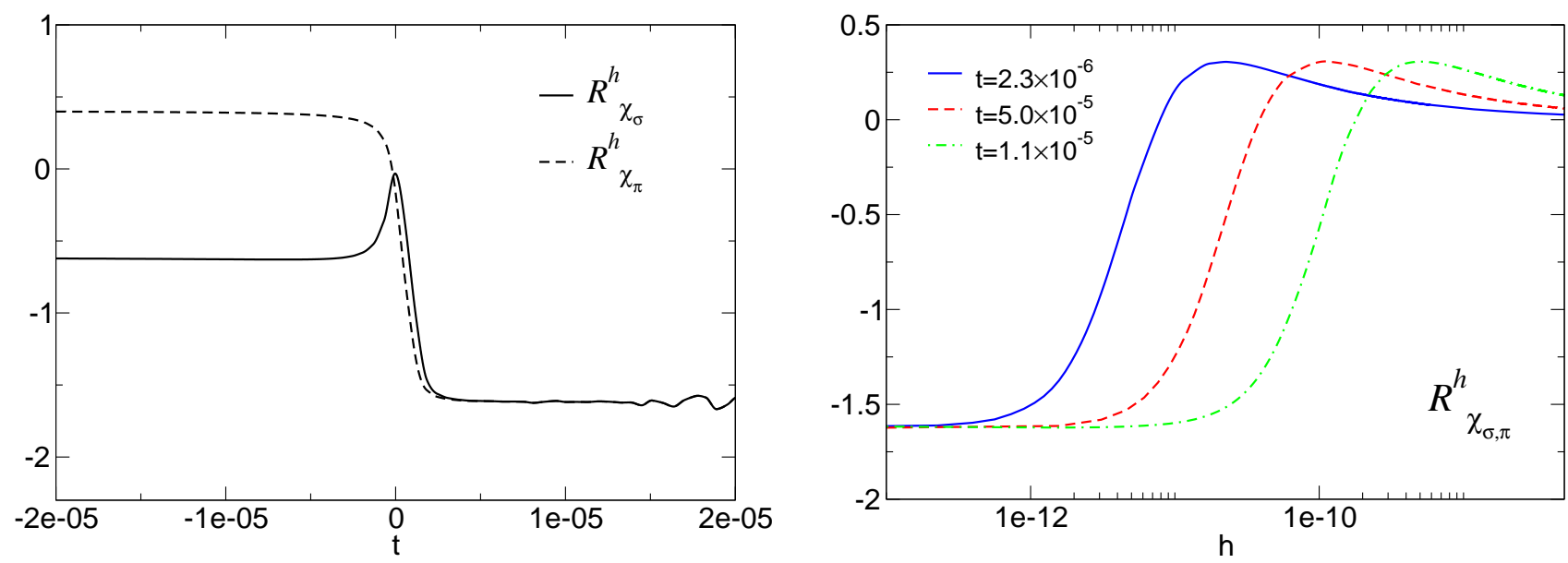

FIG. 8: The ratios $R_{\chi_{\pi, \sigma}}^{t}$ as functions of $t$ for a small $h$ (left panel) and as functions of $h$ at three values of the temperature in the symmetric phase $(t>0)$ (right panel).

as well as the properties of the Widom-Griffiths scaling function $f(x)$ discussed above, one finds

$$
\lim _{h \rightarrow 0} \Delta(t, h)= \begin{cases}1, & t>0, \\ 1 / \delta, & t=0, \\ 0, & t<0 .\end{cases}
$$

Consequently, the ratio $\Delta(t, h)$ can be used to directly extract the value of the critical exponent $\delta$, without resorting to logarithmic fits. Moreover, this ratio simultaneously reveals the position of the critical point, since in the scaling regime, all $\Delta(t, h)$, plotted as functions of $t$ for different $h$, cross at $t=0$.

In Fig. 9-left we show $\Delta(t, h)$ as a function of $t$ for four values of $h$. As expected all lines cross at $t=0$, yielding the value of the critical exponent $\delta \approx 4.91$. This value is in good agreement with the one obtained above, using a logarithmic fit to the dependence of the order parameter on $h$.

In the right panel of Fig. 9 we show the scaling of $\Delta$ as a function of $h$, for fixed $t>0$. In the chiral limit, that is for $h=0$, the longitudinal and transverse susceptibilities coincide above the critical temperature. Consequently $\Delta=1$ in the limit $h \rightarrow 0$, as shown in the plot. In the opposite limit, i.e. for $h \rightarrow \infty$, we find $\Delta=1 / \delta$. The coincidence of the two limits $h \rightarrow \infty$ and $t \rightarrow 0$ follows from the explicit form for this ratio in terms of the scaling equation of state (28), $\Delta=1 / \delta-(z / \beta \delta)\left(F_{h}^{\prime}(z) / F_{h}(z)\right)$. Since the limits $t \rightarrow 0$ for finite $h$ and $h \rightarrow \infty$ for finite $t$ both imply $z=t h^{-1 / \beta \delta} \rightarrow 0$, the result $\Delta=1 / \delta$ is obtained in both cases.

\section{Correlation lengths and their critical behavior}

A correlation length $\xi$ governs the exponential decay of the corresponding correlation function with increasing separation. The critical behavior of correlation lengths is an important characteristic of phase transitions. At the critical point of a second-order phase transition, the order-parameter correlation length diverges, heralding the onset of a long-range order.

The decay of a correlation function is controlled by the relevant mass scale in the system. In a chiral model, such as the one considered here, there are two such scales, the transverse mass connected with the pion and the longitudinal mass related to the sigma field. Consequently, like in the case of magnetization, one introduces a transverse and a longitudinal correlation length $\xi_{\pi}$ and $\xi_{\sigma}$. In the critical region for $t>0$, the correlation lengths are directly related to the corresponding susceptibilities [7, 41]

$$
\chi_{l} \sim \xi_{l}^{2}, \chi_{t} \sim \xi_{t}^{2} .
$$

Consequently, in the chiral quark-meson model, the correlation lengths are determined by the pion and sigma masses

$$
\xi_{\sigma} \sim \frac{1}{m_{\sigma}}, \quad \xi_{\pi} \sim \frac{1}{m_{\pi}} .
$$

In the broken symmetry phase, the soft transverse modes, which renormalize the longitudinal susceptibility in a non-trivial way, as shown in Eq. 49, also profoundly influence the longitudinal correlation length 42]. This modifies the relation between the correlation length and the susceptibility in the longitudinal channel for $t<0$. We do not address this problem here.

In Fig. 10 we show the correlation lengths, $\xi_{\sigma}$ and $\xi_{\pi}$, at the critical point $t=0$ as functions of the external field $h$. As expected both the longitudinal and transverse correlation lengths diverge as $h \rightarrow 0$. The divergence of $\xi_{\sigma}$ reflects the softening of order parameter fluctuations as the critical point is approached, while that of $\xi_{\pi}$ signals the appearance of Goldstone bosons at the second order phase transition.

The scaling of the correlation length in the critical region is obtained from the scaling function (28) and the 

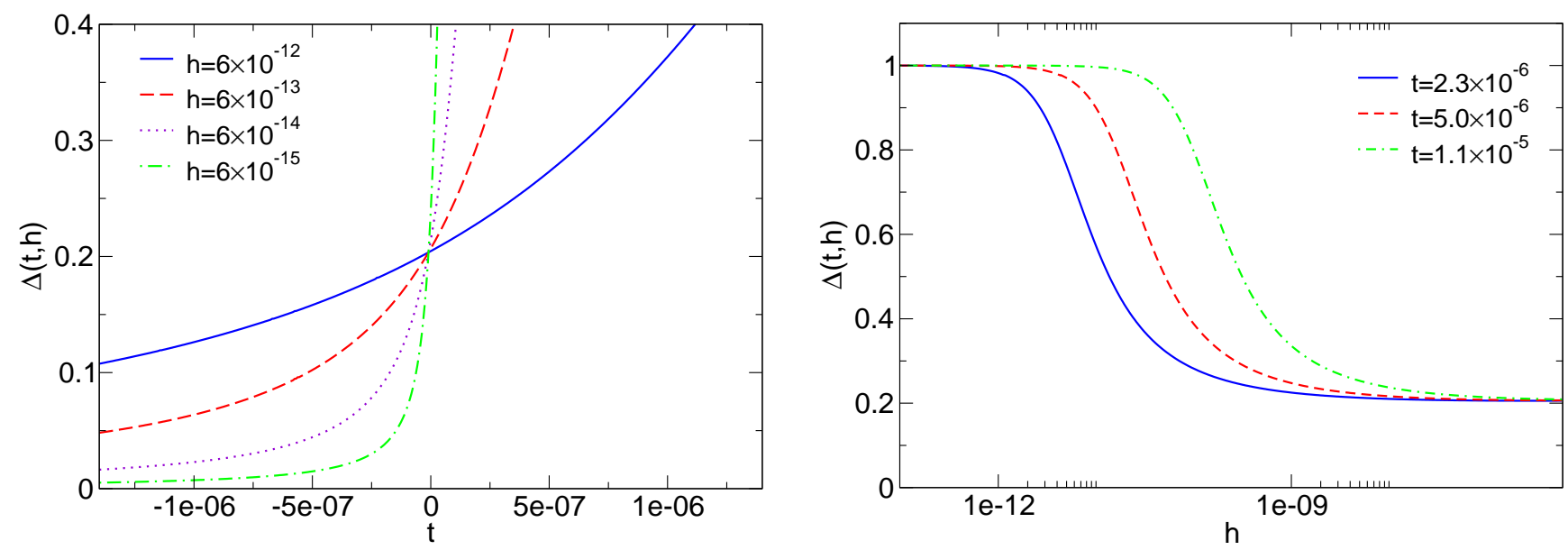

FIG. 9: The ratio $\Delta(t, h)$ defined in Eq. (53) as a function of the reduced temperature $t$ and the external field $h$. In the left-hand figure the curves cross in the point $(0,1 / \delta)$.

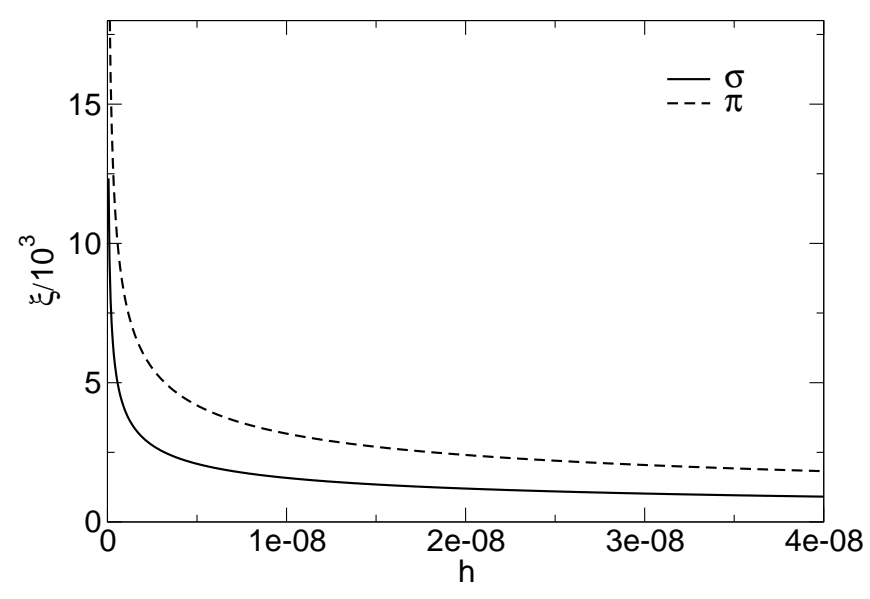

FIG. 10: The correlation lengths $\xi_{\sigma}$ and $\xi_{\pi}$ at the critical point $t=0$ as functions of the external field $h$.

relation (55). On the critical line $(t=0, h \rightarrow 0)$, the transverse and longitudinal correlation length exhibit the same singularity, controlled by the exponent $\nu_{c}$ [7]

$$
\xi_{\sigma, \pi} \sim h^{-\nu_{c}} .
$$

Their ratio, $\xi_{\pi} / \xi_{\sigma}$, is at $t=0$ approximately equal to 2 (see Fig. 10). Thus, the value expected for this ratio in the broken symmetry phase 42 is obtained also on the critical line, in agreement with [7].

In the symmetric phase $(t>0)$, the correlation lengths $\xi_{\sigma}$ and $\xi_{\pi}$ coincide and the scaling with $t$ is governed by the critical exponent $\nu$

$$
\xi_{\sigma, \pi} \sim t^{-\nu} .
$$

In Fig. 11 we show the scaling behavior of the correlation lengths on the critical line and in the symmetric phase. A fit of the critical exponents yields $\nu_{c}=0.396$ and $\nu=$ 0.787 , respectively.
Our results for the various critical exponents are summarized in Table II We give the values obtained in the logarithmic fits shown in Figs. 26] and 11, which we deem to be more accurate than the other determinations. The value for the coefficient $\alpha$ is obtained by using the hyperscaling relation (33). Our results are compared with recent lattice results and mean-field theory exponents for the $O(4)$ model. The mean-field values clearly deviate from the FRG and lattice results, showing the importance of fluctuations near the critical point. Our exponents, obtained with the FRG approach, are in good agreement with the Monte Carlo lattice results [26] in spite of the fact, that we have neglected the anomalous dimension in the flow equations.

The finding that the anomalous dimension is fairly unimportant for the critical behavior, is consistent with the relatively small value of the corresponding critical exponent $\eta$ in the $O(4)$ universality class. In spite of the fact that we neglect momentum dependent couplings in the RG flow, a fairly reasonable value for the critical exponent $\eta$, can be extracted by means of the hyperscaling relation

$$
\delta=\frac{d+2-\eta}{d-2+\eta}
$$

Using our value for $\delta$, we find $\eta \approx 0.031$, which should be compared with the lattice result, $\eta=0.0254$ [26]. We note, however, that using the middle scaling relation in (32) to determine $\eta$ fails; the resulting value is small and negative. This illustrates the uncertainty introduced by the leading order derivative expansion.

\section{SUMMARY AND CONCLUSIONS}

We have discussed the critical properties of the chiral quark-meson model at the finite-temperature phase 

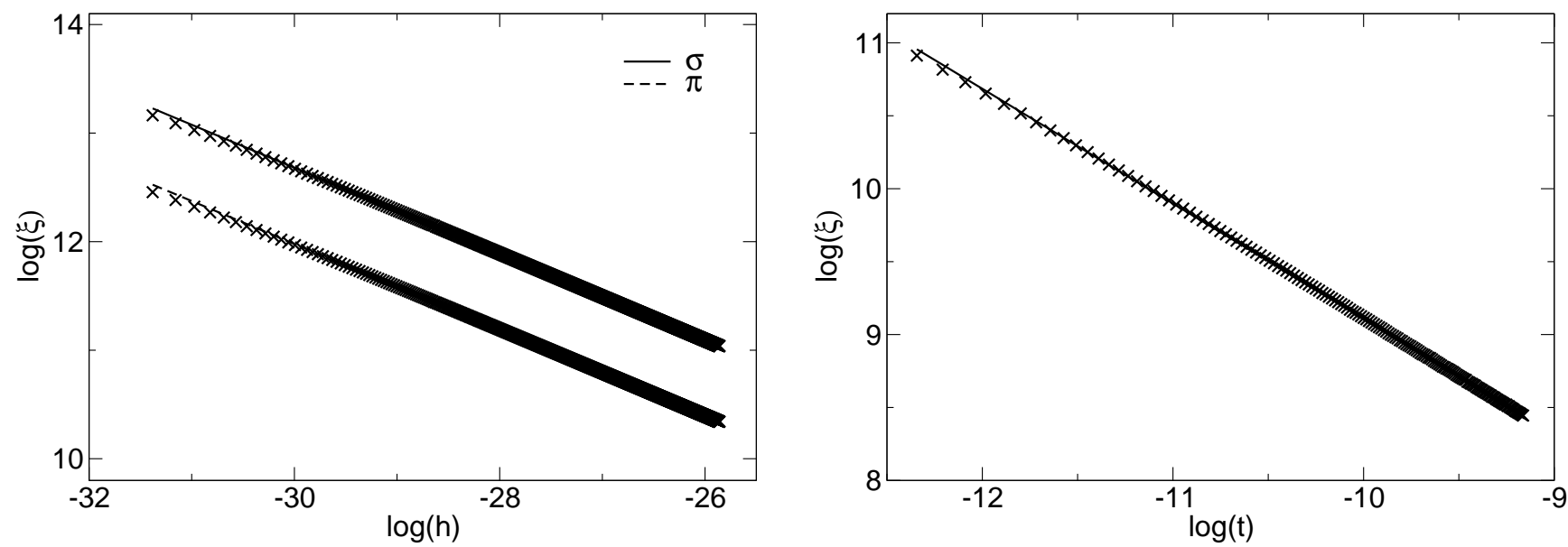

FIG. 11: The correlation lengths $\xi_{\sigma}$ and $\xi_{\pi}$ as functions of $h$ at $t=0$ (left) and as functions of $t$ at $h=0$ (right). As discussed in the text, the scaling of the correlation lengths is governed by the critical exponents $\nu_{c}$ and $\nu$. Note that the normalization of the correlation lengths differ from that used in Fig. 10.

TABLE I: The critical exponents of the $O(4)$ model obtained in lattice calculations, in the mean-field approximation and in the present FRG approach.

\begin{tabular}{lcccccc}
\hline \hline & $\beta$ & $\delta$ & $\gamma$ & $\nu$ & $\nu_{c}$ \\
\hline$O(4)$ lattice [26] & 0.3836 & 4.851 & 1.477 & 0.7479 & $0.4019^{a}$ & -0.244 \\
Mean Field & 0.5 & 3 & 1 & 0.5 & $1 / 3$ & 0 \\
FRG (our work) & 0.402 & 4.818 & 1.575 & 0.787 & 0.396 & $-0.361^{b}$ \\
\hline \hline
\end{tabular}

${ }^{a}$ Obtained using the definition of $\nu_{c}$ (31).

${ }^{b}$ Obtained using the scaling relation

transition. The focus was on the universal scaling behavior of various physical quantities. Fluctuations and nonperturbative effects were accounted for by employing the Functional Renormalization Group (FRG) method. We derived the flow equation for the scale dependent thermodynamic potential at finite temperature and chemical potential in the presence of an external field. The flow equation was then solved numerically, by employing a truncated polynomial expansion around the minimum of the effective action. We have shown that the choice of regulator functions (11) and (13) leads to transparent expressions and reliable numerical results. In this work, we have neglected the bosonic and fermionic wave function renormalization and the running of the Yukawa coupling. We argue that, in the critical region, these effects lead to sub-leading corrections to the scaling behavior.

We have computed various thermodynamical quantities that exhibit critical behavior near the chiral phase transition. In particular, we have analyzed the scaling behavior of the chiral order parameter, its longitudinal and transverse susceptibilities as well as the correlation lengths in the chiral limit and in the presence of an external symmetry breaking field. We found that the chiral order parameter and its susceptibilities scale near the chiral phase transition following the universal scaling be- havior of the singular part of the free energy. Here we have extracted the corresponding critical exponents at the critical point and at the coexistence line and have also analyzed the scaling of the pseudocritical temperature and the maxima of the longitudinal susceptibility in an external field.

We have also analyzed the effective critical exponents, which govern the leading scaling behavior above and below the critical temperature for a system in an external symmetry breaking field. In this context, the longitudinal and transverse susceptibilities are of particular interest, since they diverge at the coexistence line. The effective critical exponents obtained numerically, are in full agreement with the Widom-Griffiths form of the magnetic equation of state.

The critical exponents obtained in this work within the quark meson model by employing the FRG method, are in a very good agreement with the recent lattice results for the $O(4)$ spin system. This confirms the expectation that the chiral phase transition of the 2-flavor effective chiral Lagrangian belongs to the $O(4)$ universality class. Furthermore, our results demonstrate the power of the FRG approach and the validity of the truncation approximation to the flow equation. This approach offers an efficient framework for describing fluctuations and 
non-perturbative long-distance dynamics near the chiral phase transition.

We note in closing, that the critical region (the region of true non-trivial critical behavior) of the chiral quarkmeson model turns out to be very small in our calculation. This is reflected in the very small values for $t$ and $h$ needed in order to obtain stable results in the logarithmic fits of the critical exponents. Our finding is consistent with the suppression of the Ginsburg region [43] in $O(N)$ models for large $N$. However, because the size of the critical region is not universal, the quantitative implications for the chiral transition in QCD are uncertain.

\section{Acknowledgment}

We thank Jürgen Engels, Frithjof Karsch and BerndJochen Schaefer for useful comments and stimulating discussions. K.R. partial support from the Polish Ministry of Science and the Deutsche Forschungsgemeinschaft (DFG) under the Mercator Programme. B.S. gratefully acknowledges financial support from the Helmholtz Research School on Quark Matter Studies.
[1] M. Cheng et al., Phys. Rev. D77, 014511 (2008).

[2] R. D. Pisarski and F. Wilczek, Phys. Rev. D29, 338 (1984).

[3] K. Rajagopal and F. Wilczek, Nucl. Phys. B399, 395 (1993).

[4] F. Parisen Toldin, A. Pelissetto and E. Vicari, JHEP 0307, 029 (2003).

[5] J. Engels, S. Holtmann, T. Mendes and T. Schulze, Phys. Lett. B514, 299 (2001).

[6] J. Engels and T. Mendes, Nucl. Phys. B572, 289 (2000).

[7] J. Engels, L. Fromme and M. Seniuch, Nucl. Phys. B675, 533 (2003).

[8] By RBC-Bielefeld collaboration (Frithjof Karsch for the collaboration). Oct 2008. 4pp. Presented at International Conference on Strong and Electroweak matter (SEWM 2008), Amsterdam, The Netherlands, 26-29 Aug 2008. e-Print: arXiv:0810.3078 [hep-lat].

[9] B. J. Schaefer and J. Wambach, Nucl. Phys. A757, 479 (2005).

[10] S. Roessner, C. Ratti and W. Weise, Phys. Rev. D75, 034007 (2007).

[11] B. J. Schaefer and J. Wambach, Phys. Rev. D75, 085015 (2007).

[12] C. Sasaki, B. Friman and K. Redlich, Phys. Rev. D77, 034024 (2008). Phys. Rev. Lett. 99, 232301 (2007). Phys. Rev. D75, 074013 (2007).

[13] S. K. Ma, Rev. Mod. Phys. 45, 589 (1973).

[14] J. Berges, N. Tetradis and C. Wetterich, Phys. Rept. 363, 223 (2002).

[15] D. U. Jungnickel and C. Wetterich, Phys. Rev. D53, 5142 (1996).

[16] J. Berges, D. U. Jungnickel and C. Wetterich, Eur. Phys. J. C13, 323 (2000).

[17] J. Meyer, G. Papp, H. J. Pirner and T. Kunihiro, Phys. Rev. C61, 035202 (2000).

[18] J. Braun, K. Schwenzer and H. J. Pirner, Phys. Rev. D70, 085016 (2004).

[19] J. Berges, D. U. Jungnickel and C. Wetterich, Phys. Rev. D59, 034010 (1999).

[20] N. Tetradis, Nucl. Phys. A726, 93 (2003).
[21] B. J. Schaefer and H. J. Pirner, Nucl. Phys. A660, 439 (1999).

[22] J. Braun and B. Klein, Phys. Rev. D77, 096008 (2008).

[23] A. Onuki, Phase transition dynamics (Cambridge University Press, Cambridge, 2002)

[24] E. K. Riedel and F. J. Wegner, Phys. Rev. B9, 924 (1974)

[25] G. Boyd, J. Fingberg, F. Karsch, L. Karkkainen and B. Petersson, Nucl. Phys. B376, 199 (1992).

[26] K. Kanaya and S. Kaya, Phys. Rev. D51, 2404 (1995).

[27] C. Wetterich, Phys. Lett. B301, 90 (1993).

[28] T. R. Morris, Int. J. Mod. Phys. A9, 2411 (1994).

[29] U. Ellwanger, Z. Phys. C62, 503 (1994).

[30] D. F. Litim, Phys. Rev. D64, 105007 (2001).

[31] J.-P. Blaizot, A. Ipp, R. Méndez-Galain and N. Wschebor, Nucl. Phys. A784, 376 (2007)

[32] K.G. Wilson and J.B. Kogut, Phys. Rept. 12, 75 (1974).

[33] D. J. Wallace and R. K. P. Zia, Phys. Rev. B12, 5340 (1975).

[34] L. D. Landau and E. M. Lifshitz, Statistical Physics, (Pergamon Press, Oxford, 1980)

[35] R. B. Griffiths, Phys. Rev. 158, 176 (1967).

[36] E. Brezin, D. J. Wallace and K. G. Wilson, Phys. Rev. B7, 232 (1973).

[37] A. Pelissetto and E. Vicari, Phys. Rept. 368, 549 (2002).

[38] E. Brezin and D. J. Wallace, Phys. Rev. B7, 1967 (1973).

[39] F. Karsch and E. Laermann, Phys. Rev. D50, 6954 (1994).

[40] A. Kocic, J. B. Kogut and M. P. Lombardo, Nucl. Phys. B398, 376 (1993).

[41] M. E. Fisher, V. Privman, Phys. Rev. B32, 447 (1985).

[42] M.E. Fisher, M.N. Barber and D. Jasnow, Phys. Rev. A8, 1111 (1973)

[43] J.B. Kogut, M.A. Stephanov and C.G. Strouthos, Phys. Rev. D58, 096001 (1998)

[44] The scalar condensate is proportional to the quark condensate $\langle\bar{q} q\rangle$.

[45] We note that $\partial \Omega_{k} / \partial \rho$ vanishes, due to the gap equation (17), so that $d \Omega_{k} / d k=\partial \Omega_{k} / \partial k$. 